\title{
On the Existence of Solutions to Einstein's Equation With Non-Zero Bondi News
}

\author{
Abhay Ashtekar* and Tevian Dray ${ }^{\star \star}$ \\ Département de Physique, Université de Clermont-Fd., F-63170 Aubière, France
}

\begin{abstract}
It is shown that the $C$-metric (with parameters chosen to lie in suitable intervals) admits a conformal completion such that the space of generators of null infinity, $\mathscr{I}$, is a 2 -sphere. This structure of $\mathscr{I}$ is both necessary and sufficient for the analysis of gravitational radiation in exact general relativity. Bondi news (as well as the electromagnetic radiation field, in the charget case) is examined and found to be non-zero. Thus the issue of existence of exact solutions to the Einstein (and Einstein-Maxwell) equations admitting radiation (in the sense of Bondi, Sachs, and Penrose) is resolved. In addition, the analysis clarifies the sense in which the vacuum $C$-metric represents the gravitational field of two accelerating black-holes.
\end{abstract}

\section{Introduction}

Penrose's [1] null infinity, $\mathscr{I}$, provides an elegant, geometrical method to impose the boundary conditions that are believed to be appropriate in problems involving gravitational radiation. Furthermore, the single assumption that a given spacetime admits a $\mathscr{I}$ whose space of generators is a 2 -sphere enables one to obtain a rich structure of direct physical significance ${ }^{1}$ : one can introduce, in a precise fashion, various asymptotic fields, prove peeling theorems, show that the flux of energy carried away by gravitational waves is positive, compute the Bondi 4-momentum at any retarded instant of time, etc. Consequently, over the years, the notion of $\mathscr{I}$ has become crucial to the gravitational radiation theory: essentially every rigorous result conerning radiation in exact general relativity assumes, at least implicitly, the existence of $\mathscr{I}$. Furthermore, without recourse to $\mathscr{I}$, one can

* Address as of September, 1980: Physics Department, Syracuse University, Syracuse, NY 13210

$\star \star$ Address as of January, 1981: Mathematics Department, University of California Berkley, Ca 94720

1 If the space of generators fails to be a 2-sphere, however, one can do very little: one can introduce neither the news tensor nor the Bondi 4-momentum. For details, see e.g. [1] or [2] 
not even formulate basic ideas such as "the absence of incoming radiation in a given system". The notion has also played a key role in a variety of global issues in general relativity. Examples of these are: analysis of singularities, cosmic censorship hypothesis, black hole theory, $S$-matrix description of classical and quantum zero rest-mass fields and $H$-space theory.

One would therefore like to have strong evidence in support of the belief that the assumption of existence of $\mathscr{I}$ is not an excessively stringent one. For the class of stationary space-times, the evidence is resonably strong. For example, one can show explicitly that Schwarzschild, Kerr and certain Weyl solutions are asymptotically flat at null infinity in the desired sense. In the general context, however, the evidence is surprisingly weak: no non-stationary solution of Einstein's equation has been shown to admit a $\mathscr{I}$ which is topologically $S^{2} \times R$ ! Thus, one is faced with a curious situation: although a rich, general framework describing gravitational radiation has been available in the exact theory for over fifteen years, not a single example is known to which the framework is applicable. The only concrete evidence in the general case comes from the stability analyses of Lerner and Porter [3] and Geroch and Xanthopoulos [4]. The first of these provides a statement of "kinematical stability": it shows that in a certain topology on the space of all Lorentz metrics on $R^{4}$, asymptotically simple space-times [1] form an open neighborhood of Minkowski space. The second analysis provides a more powerful statement of "dynamical stability": it shows that the notion of asymptotic simplicity is preserved to first order by gravitational perturbations with initial data of compact support on a Cauchy slice. These results - particularly the second - do reinforce one's belief that existence of $\mathscr{I}$ is a reasonable hypothesis. However, they do not quite resolve the issue of existence of asymptotically flat radiating solutions. For, the field equations are completely ignored in [3] and one has very little intuition about the cumulative effects, over infinite time ${ }^{2}$, of the nonlinear contributions ignored in [4]. To improve this situation, a completely new approach has recently been adopted by Friedrich [5]. He tries to solve the characteristic initial value problem for Einstein's equation using $\mathscr{I}$ as one of the null surfaces on which the data is specified. The issue of uniqueness of solutions has already been resolved. The issue of existence, on the other hand, is open and appears to be a highly non-trivial one. The resolution of this issue will of-course give a strong evidence - perhaps the strongest one can hope for - in favor of one's belief concerning null infinity.

In this paper we follow yet another avenue. We shall analyse certain known solutions of Einstein and Einstein-Maxwell equations - the $C$-metrics [6-8] with parameters restricted to suitable ranges - and show explicitly that they admit a conformal completion in which the space of generators of $\mathscr{I}$ is a 2 -sphere. This property enables one to introduce various notions - such as the BMS Lie algebra and the Bondi news - of interest to the gravitational radiation theory and to compute quantities - such as the Bondi 4-momentum and the fluxes of energymomentum and angular momentum - of physical significance. An explicit computation will show that the electromagnetic radiation field on $\mathscr{I}$ is non-zero in

2 This point was emphasized by B. G. Schmidt during the Cincinnati meeting on Asymptotic Structure of Space-time 
the electrovac case and the Bondi news is non-zero in all cases. Thus, the analysis will resolve the issue of existence of exact solutions to the Einstein and EinsteinMaxwell equations which admit radiation in the sense of Bondi, Sachs and Penrose. The discussion may also be helpful in the investigation of equations of motion since, as we shall see, the solutions that we analyse represent the gravitational (and, in the charged case, also the electromagnetic) field of two accelerating particles. Furthermore, with an appropriate choice of parameters, these particles appear to be black holes. Hence, the analysis may also be of interest to groups investigating black hole collisions using computer methods.

The approach has two major drawbacks. First, since we focus on specific examples rather than on general theorems, the analysis does not shed any light on whether or not the assumption of existence of $\mathscr{I}$ is likely to be satisfied in generic, physically reasonable situations; we obtain only a 2-parameter family of vacuum and a 3-parameter family of electrovac asymptotically flat space-times. Furthermore, as is easy to see using symmetry arguments, the total (ADM) 4-momentum of every space-time to be considered vanishes identically; the spacetimes are very special indeed in this respect. Therefore, to resolve the issue of whether or not a "sufficient number" of asymptotically flat radiating solutions exist, one must await, e.g., the completion of the Friedrich program. The second drawback is that none of the space-times to be analysed admits a $\mathscr{I}$ with complete generators. While the completeness property plays essentially no role in the gravitational radiation theory, it is important in applications of the $\mathscr{I}$-formalism to the black hole theory, the cosmic censorship hypothesis and the $S$-matrix theory of zero rest-mass fields. The present discussion makes no contribution to the issue of existence of radiationg solutions with complete $\mathscr{I}$.

The material is organized as follows. Section 2 is devoted to preliminaries: definitions of asymptotic flatness are introduced and the relevant properties of the $C$-metric are recalled. It turns out that, except for two 2-parameter families of electrovac solutions, the metrics possess 2-dimensional sheets of nodal singularities which complicate the global analysis considerably. Therefore, to begin with, we consider in Sect. 3 the metrics without nodes: We show that they admit $\mathscr{I}$ which is topologically $S^{2} \times R$ and have gravitational (as well as electromagnetic) radiation. These space-times are, however, geodesically incomplete and can be extended analytically. In order to bring out the geometrical significance of the various steps involved in this procedure, we restrict ourselves in Sect. 4 to the flat $C$-metric - i.e. to the case where the mass and the charge parameters are set equal to zero - and carry out the extension explicitly. In Sect. 5, we consider $C$-metrics with nodes. In this case, it is essential to carry out the extension to obtain $\mathscr{I}$ which is topologically $S^{2} \times R$ : due to the presence of nodes, the null boundary of the original, unextended space-time "misses" one generator. The extended space-time represents the field of two uniformly accelerating particles. In Sect. 6, we point out that there is a sense in which the two particles may be regarded as black holes for a certain range of parameters. However, the system is not isolated: since the metric is time-symmetric, and since there is outgoing radiation on future null infinity, there is also incoming radiation on past null infinity. Furthermore, the presence of the nodal singularity - a 2-dimensional time-like sheet which can be placed either "in between" the two holes or in the region between each hole and infinity - suggests 
that there exist struts or strings preventing a head-on collision between the two particles $^{3}$.

\section{Preliminaries}

We begin with certain definitions. By space-time $\left(\hat{M}, \hat{g}_{a b}\right)$, we shall mean a $C^{\infty}$ manifold $\hat{M}$ (possibly with boundary) equipped with a $C^{\infty}$ metric $\hat{g}_{a b}$ of signature $(-+++)$. A space-time $\left(\hat{M}, \hat{g}_{a b}\right)$ will be said to be asymptotically empty and flat at null infinity if there exists a space-time $\left(M, g_{a b}\right)$ equipped with a $C^{\infty}$ function $\Omega$ and an imbedding of $\hat{M}$ into $M$ (by which we shall identify $\hat{M}$ and its image in $M$ ) such that:

i) $g_{a b}=\Omega^{2} \hat{g}_{a b}$ on $\hat{M}$;

ii) $\Omega=0$ on $\partial \hat{M} ; \nabla_{a} \Omega \neq 0$ on $\partial \hat{M}$;

iii) the manifold of orbits of the restriction of the vector field $n^{a}:=\nabla^{a} \Omega$ to $\partial \hat{M}$ is diffeomorphic to $S^{2}$; and

iv) the tensor field $\Omega^{-2} \hat{R}_{a b}$ admits a smooth limit to $\partial \hat{M}$, where $\hat{R}_{a b}$ is the Ricci tensor of $\hat{g}_{a b}$.

The boundary $\partial \hat{M}$ of $\hat{M}$ in $M$ represents null infinity and will be denoted by $\mathscr{I}$. The condition iv) is equivalent to demanding that the stress-energy tensor of $\left(\hat{M}, \hat{g}_{a b}\right)$ vanish asymptotically to order 4 in the sense of Geroch [2]. i), ii), and iv) imply that $\mathscr{I}$ is a null 3 -surface and that one can always perform a conformal rescaling such that the vector field $n^{a}$ is divergence-free on $\mathscr{I}$. Together with iii) they imply that the Weyl tensor of $g_{a b}$ must vanish on $\mathscr{I}$. This enables one to introduce on $\mathscr{I}$ various asymptotic fields of physical interest and to define Bondi 4-momentum. If conditions i) and ii) alone are satisfied, we say that $\left(\hat{M}, \hat{g}_{a b}\right)$ admits a local $\mathscr{I}$. If conditions i), ii), and iii) are satisfied, we shall say that it admits a global $\mathscr{I}$. Note that one cannot introduce the news tensor or the Bondi 4-momentum unless $\left(\hat{M}, \hat{g}_{a b}\right)$ admits a global $\mathscr{I}$. Finally, we shall say that $\left(\hat{M}, \hat{g}_{a b}\right)$ is asymptotically Minkowskian if it satisfies, in addition to i), ii), iii), and iv) the condition that, in the conformal frames in which $n^{a}$ is divergence-free, it is also a complete vector field. Thus, a space-time is asymptotically Minkowskian if it is asymptotically empty and flat at null infinity and if the generators of $\mathscr{I}$ are complete.

Next, we collect - essentially from [8] - the relevant properties of the $C$-metric. The term " $C$-metric" is used as a collective label for a 3-parameter family of solutions to Einstein-Maxwell equations. The space-time metric $\hat{g}_{a b}$ is given by:

$$
\hat{g}_{a b} d x^{a} d x^{b} \equiv d \hat{s}^{2}=r^{2}\left[-F(y) d t^{2}+F(y)^{-1} d y^{2}+G(x) d z^{2}+G(x)^{-1} d x^{2}\right],
$$

3 The $C$-metric is analysed in detail in [8] and its generalization [9] to include the twist of the Killing fields in [10]. Some of the results of these papers - particularly certain Penrose diagrams with $\mathscr{I}$ in [8] and the calculation of the Bondi 4-momentum in [10] - may seem to contradict the assertion that no non-stationary solution to Einstein's equation has been shown to admit null infinity which is topologically $S^{2} \times R$. We would therefore like to clarify the situation: that the $C$-metrics admit an appropriate $\mathscr{I}$ is an assumption in both sets of analyses. Also, as we shall see in Sect. 6, it turns out that the Penrose diagrams of [8] are not quite accurate. Furthermore, due to nodal problems, the assumption that a Newman-Unti coordinate system - global in angular variables - exists in the asymptotic region of the (unextended) field of a single particle, made in both [8] and [10] and used crucially in the computation of the Bondi 4-momentum in [10], is not likely to be satisfied 
where:

$$
r=[A(x+y)]^{-1} ; \quad F(y)=-G(-y) ; \quad G(x)=1-x^{2}-2 m A x^{3}-e^{2} A^{2} x^{4} ;
$$

and

and the Maxwell field $\hat{F}_{a b}$ by ${ }^{4}$ :

$$
m \geqq 0, \quad A>0,
$$

$$
\hat{F}_{a b}=2 e \hat{\nabla}_{[a} y \hat{\nabla}_{b]} t .
$$

The parameter $m$ is interpreted as the mass, $e$ as the charge, and $A$ as the acceleration of a particle whose gravitational and electromagnetic fields are described by the solution. The particle is "located" at $r=0$, which is a curvature singularity if at least one of $m$ and $e$ is non-zero. If $m=e=0$, the metric $\hat{g}_{a b}$ is flat. In general, $\hat{g}_{a b}$ is a solution to the electrovac equation if $e \neq 0$ and to the vacuum equation if $e=0$. The metric admits precisely two independent Killing fields, $\partial_{t}$ and $\partial_{z}$. Both are hypersurface orthogonal and they commute. (In the flat space limit, $\partial_{t}$ reduces to a boost - say, in the $\bar{z}-\bar{t}$ plane - and $\partial_{z}$ to the rotation in the $\bar{x}-\bar{y}$ plane, where $\bar{x}, \bar{y}, \bar{z}, \bar{t}$ denote the Cartesian coordinates.) $\hat{g}_{a b}$ also admits a conformal Killing tensor $\hat{K}^{a b} \equiv G(x)^{-1} z^{a} z^{b}+G(x) x^{a} x^{b}$, where $z^{a} \equiv \partial_{z}$ and $x^{a} \equiv \partial_{x}$. ( $\hat{K}^{a b}$ is, incidentally, a Killing tensor of $g_{a b}=r^{-2} \hat{g}_{a b}$.) The presence of these symmetries greatly simplifies the task of integrating all null geodesics in the spacetime. The Weyl tensor is of type (2-2).

Next, we consider coordinate ranges. Since the signature of $\hat{g}_{a b}$ is required to be $(-+++)$, we must restrict $x$ to a domain on which $G(x)$ is non-negative. We shall assume that $G(x)$ has at least two real roots and require $x$ to lie in $\left[x_{2}, x_{1}\right]$ such that $G(x)>0 \forall x \in] x_{2}, x_{1}\left[\right.$ and $G\left(x_{1}\right)=G\left(x_{2}\right)=0$. Although this restricts the parameters somewhat, it enables us to endow the $x-z$ submanifolds with the topology (and the differential structure) of $S^{2}$ which is crucial in the construction of $\mathscr{I}$. Set:

$$
\theta=\int_{x_{2}}^{x} G(x)^{-1 / 2} d x
$$

and

$$
\phi=\kappa^{-1} z, \quad \phi \in[0,2 \pi],
$$

where $\kappa$ is a (positive) constant, to be specified below. The metric on the $x-z$ submanifold now takes the form

$$
\begin{aligned}
d \hat{\sigma}^{2} & \equiv r^{2}\left[G(x) d z^{2}+G(x)^{-1} d x^{2}\right] \\
& =r^{2}\left[\varrho^{2}(\theta) \kappa^{2} d \phi^{2}+d \theta^{2}\right],
\end{aligned}
$$

where, we have set $\varrho(\theta)=\sqrt{G(x)}$. $\left(\varrho(\theta)\right.$ vanishes at $\theta=0$ and at $\theta_{0}=\left.\theta\right|_{x_{1}}$. It is positive and bounded in $\left[0, \theta_{0}\right]$.) The metric is clearly regular at points other than the roots of $\varrho, \theta=0$ and $\theta=\theta_{0}$. It is easy to verify [8] that a necessary and sufficient condition for the metric to be regular also at these roots is : $\kappa^{-1}=|\partial \varrho / \partial \theta|$ at the

4 As in all electrovac solutions, there is some freedom in the choice of $\hat{F}_{a b}$ : since the metric determines only the stress-energy tensor, one can replace $\hat{F}_{a b}$ by $\cos \alpha \hat{F}_{a b}+\sin \alpha \hat{F}_{a b}$ for any constant $\alpha$. The expression in Eq. (2.3) is singled out by demanding that the electric charge be $e$ and that the magnetic charge be zero 
roots. If this condition is not satisfied at a root, the metric has a nodal singularity there. Since we have only a single constant $\kappa$ at our disposal, we cannot avoid the nodal singularity at one of the two roots unless

$$
|\partial \varrho / \partial \theta|_{\theta=0}=|\partial \varrho / \partial \theta|_{\theta=\theta_{0}} .
$$

This condition imposes a severe restriction on the choice of parameters appearing in the quartic $G(x)$ : it is satisfied if and only if $m=0$ or $m=|e|>1 /(4 A)$. Thus, except for two 2-parameter families of electrovac solutions, the metric inherits a nodal singularity either at $\theta=0$ or at $\theta=\theta_{0}$. The only vacuum metric without nodes is therefore the flat one, corresponding to $m=e=0$. [Note, incidentically, that the condition $m=0$ or $m=|e|>1 /(4 A)$ implies that $G$ - and hence also $F$ - has exactly two real roots.] In the general case, we shall set $\kappa^{-1}=|\partial \varrho / \partial \theta|_{\theta=0}$, so that the node will appear at $\theta=\theta_{0}$. Let us now consider the $y-t$ submanifolds. Since there is a curvature singularity at $r=0$, we restrict ourselves to the region $0<r<\infty$. This corresponds to choosing the range $-x<y<\infty$ and $-\infty<t<\infty$. In this range, however, the metric is ill-behaved at the roots of $y$. These "coordinate singularities" can be removed by introducing $w$ via :

$$
w=t+\int F(y)^{-1} d y
$$

and, by eliminating $t$ in favor of $w$ in the expression of the metric. We have, from Eq. (2.1), (2.5), and (2.7):

$$
d \hat{s}^{2}=r^{2}\left[-F(y) d w^{2}+2 d w d y+d \theta^{2}+\kappa^{2} \varrho(\theta)^{2} d \phi^{2}\right]
$$

with :

$$
\begin{gathered}
-\infty<w<\infty, \quad \phi \in[0,2 \pi] \\
\theta \in\left[0, \theta_{0}\right], \quad-x \equiv-x_{2}-\int_{0}^{\theta} \varrho(\theta) d \theta<y<\infty .
\end{gathered}
$$

In this range, the metric is regular everywhere except that a time-like, 2-dimensional sheet of nodal singularity appears at $\theta=\theta_{0}$ unless $m=0$ or $m=|e|$ $>1 / 4 A$.

Finally, we give the expression of the curvature tensor of the metric of Eq. (2.8). The Ricci tensor is given by

$$
\hat{R}_{a b}=(e / r)^{2}\left[F(y) \hat{\nabla}_{a} w \hat{\nabla}_{b} w-2 \hat{\nabla}_{(a} w \hat{\nabla}_{b)} y+\hat{\nabla}_{a} \theta \hat{\nabla}_{b} \theta+\kappa^{2} \varrho(\theta)^{2} \hat{\nabla}_{a} \phi \hat{\nabla}_{b} \phi\right]
$$

and the Weyl tensor by

$$
\begin{aligned}
& \hat{C}_{a b c d}=4 r P\left\{-2 \hat{\nabla}_{[a} w \hat{\nabla}_{b]} y \hat{\nabla}_{[c} w \hat{\nabla}_{d]} y+F(y) \hat{\nabla}_{[a} w \hat{\nabla}_{b]} \theta \hat{\nabla}_{c} w \hat{\nabla}_{d]} \theta\right. \\
& +\kappa^{2} F(y) \varrho(\theta)^{2} \hat{\nabla}_{[a} w \hat{\nabla}_{b]} \phi \hat{\nabla}_{[c} w \hat{\nabla}_{d]} \phi+2 \kappa^{2} \varrho(\theta)^{2} \hat{\nabla}_{[a} \theta \hat{\nabla}_{b]} \phi \hat{\nabla}_{[c} \theta \hat{\nabla}_{d]} \phi \\
& -\hat{\nabla}_{[a} w \hat{\nabla}_{b]} \theta \hat{\nabla}_{[c} y \hat{\nabla}_{d]} \theta-\hat{\nabla}_{[a} y \hat{\nabla}_{b]} \theta \hat{\nabla}_{[c} w \hat{\nabla}_{d]} \theta \\
& \left.-\kappa^{2} \varrho(\theta)^{2} \hat{\nabla}_{[a} w \hat{\nabla}_{b]} \phi \hat{\nabla}_{[c} y \hat{\nabla}_{d]} \phi+\kappa^{2} \varrho(\theta)^{2} \nabla_{[a} y \hat{\nabla}_{b]} \phi \hat{\nabla}_{[c} w \hat{\nabla}_{d]} \phi\right\},
\end{aligned}
$$

where,

$$
P=m-e^{2} A(y-x) \equiv m-e^{2} A\left(y-\int_{0}^{\theta} \varrho(\theta) d \theta-x_{2}\right) .
$$


These expressions will enable us to show in the next section that the $C$-metric is asymptotically empty in the desired sense and that it admits gravitational radiation.

\section{Metrics Without Nodes}

In this section, we restrict ourselves to the two 2-parameter families of solutions without nodes - with $m=0$ or $m=|e|>1 /(4 A)$ - and show that these space-times are asymptotically empty and flat at null infinity. This construction will resolve the existence of radiating electrovac solutions with global $\mathscr{I}$.

Let $M$ be the manifold with boundary coordinatized by $w, y, \theta, \phi$ where the ranges of $w, \theta$, and $\phi$ are the same as in Eq. (2.8) but where the range of $y$ is extended to allow points $y=-x$; i.e., $r=\infty:-x_{2}-\int_{0}^{\theta} \varrho(\theta) d \theta \leqq y<\infty$. Clearly, $\hat{M}$ is naturally imbedded in $M$. Set, on $M$,

$$
\Omega=r^{-1}=A(y+x)=A\left(y+x_{2}+\int_{0}^{\theta} \varrho(\theta) d \theta\right)
$$

and,

$$
g_{a b} d x^{a} d x^{b} \equiv d s^{2}=\Omega^{2} d \hat{s}^{2}=-F(y) d w^{2}+2 d w d y+\kappa^{2} \varrho(\theta)^{2} d \phi^{2}+d \theta^{2} .
$$

Clearly, $g_{a b}$ is $C^{\infty}$ and non-degenerate everywhere on $M$ (including the zeros of $F$ ), and has signature $(-+++)$. The boundary $\mathscr{I}$ is given by the surface $\Omega=0$. This is a 3-dimensional submanifold of $M$, coordinatized by, say, $w, \theta, \phi$. Hence it has the topology of $S^{2} \times R$. Since $\mathrm{d} \Omega=A(d y+\varrho(\theta) d \theta)$, it vanishes nowhere on $\mathscr{I}$. Next, it follows from Eq. (2.9) that

$$
\Omega^{-2} \hat{R}_{a b} \equiv e^{2}\left(F(y) \nabla_{a} w \nabla_{b} w-2 \nabla_{(a} w \nabla_{b)} y+\nabla_{a} \theta \nabla_{b} \theta+\kappa^{2} \varrho(\theta)^{2} \nabla_{a} \phi \nabla_{b} \phi\right)
$$

admits a smooth limit to $\mathscr{I}$. Thus, conditions i), ii), and iv) of the definition of asymptotic flatness are satisfied. It remains only to show that the manifold of orbits of $n^{a} \equiv \nabla^{a} \Omega$ is diffeomorphic to $S^{2}$. We have, in the $w, y, \theta, \phi$ chart:

$$
n^{a}=g^{a b} \nabla_{b} \Omega \equiv A\left(\partial_{w}+F(y) \partial_{y}+\varrho(\theta) \partial_{\theta}\right)
$$

Hence, although $\mathscr{L}_{n} \phi=0, L_{n} \theta=A \varrho(\theta) \neq 0$; the "angular" coordinate $\theta$ does not admit an unambiguous projection to the manifold $\mathscr{S}$ of orbits of $n^{a}$. Therefore, the fact that $\theta-\phi$ surfaces are 2 -spheres does not by itself imply that the space of generators of $\mathscr{I}$ is $S^{2}$. Note, however, that $\mathscr{L}_{n} w=A ; w$ is an affine parameter for the vector field $n^{a}$. Consequently, the surface $w=w_{0}$ intersects each generator of $\mathscr{I}$ at precisely one point, i.e. provides a cross-section of $\mathscr{I}$. This cross-section is labelled by $\theta, \phi$, i.e., is a 2 -sphere. Define a function $\psi(\theta, w)$ on $\mathscr{I}$ by: $\mathscr{L}_{n} \psi=0$ and $\psi\left(\theta, w_{0}\right)=\theta$. Then, we can use $\psi$ and $\phi$ as coordinates on $\mathscr{S}$. Clearly, $\mathscr{S}$ is diffeomorphic to the cross-section $w=w_{0}$, and hence to $S^{2}$. Thus $\left(\hat{M}, \hat{g}_{a b}\right)$ is asymptotically empty and flat at null infinity.

We now analyse the structure intrinsic to $\mathscr{I}$ and give explicit expressions of the various fields involved. We shall regard $\mathscr{I}$ as a 3 -manifold in its own right, 
coordinatized by $w, \theta$, and $\phi$. The degenerate metric $q_{a b}$ on $\mathscr{I}$ - the pull-back of $g_{a b}$ - is given by

$$
q_{a b} d x^{a} d x^{b} \equiv d s^{2}=\varrho(\theta)^{2} d w^{2}-2 \varrho(\theta) d \theta d w+d \theta^{2}+\kappa^{2} \varrho(\theta)^{2} d \phi^{2} .
$$

The vector field $n^{a}$, when expressed as a linear combination of the triad $\bar{\partial}_{w}, \bar{\partial}_{\theta}, \bar{\partial}_{\phi}$ intrinsic to $\mathscr{I}$, becomes:

$$
n^{a} \equiv A\left(\bar{\partial}_{w}+\varrho(\theta) \bar{\partial}_{\theta}\right),
$$

where $\bar{\partial}$ denotes partial derivatives within $\mathscr{I}$ in the given coordinate system. (The fact that $q_{a b} n^{b}=0$ on $\mathscr{I}$ gives us the "direction field" $n^{a}$, and $L_{n} w=A$ gives us the normalization.) The fact that $\psi(w, \theta)$ satisfies, on $\mathscr{I}, L_{n} \psi=0$ and $\psi\left(\theta, w_{0}\right)=\theta$, implies that

$$
\frac{d \psi}{\varrho(\psi)}=-d w+\frac{d \theta}{\varrho(\theta)}
$$

holds everywhere on $\mathscr{I}$. Hence, in the $w, \psi, \phi$ chart on $\mathscr{I}, q_{a b}$ is given by

$$
d s^{2}=[\varrho(\theta) / \varrho(\psi)]^{2}\left(d \psi^{2}+\kappa^{2} \varrho(\psi)^{2} d \phi^{2}\right),
$$

where $\theta \equiv \theta(w, \psi)$ is now regarded as a function of $w$ and $\psi$. Note that, although $\varrho(\theta)$ and $\varrho(\psi)$ have zeros, $\varrho(\theta) / \varrho(\psi)$ is everywhere regular and non-zero. [Proof: since $\mathscr{L}_{n} \theta=A \varrho(\theta), \varrho(\theta)$ vanishes only along two entire generators. Since on the cross-section $w=w_{0}, \psi=\theta$, and since $\mathscr{L}_{n} \psi=0$ everywhere on $\mathscr{I}, \varrho(\psi)=0$ precisely along the same two generators. Consider one of these generators, say, $\theta=\psi=0$. Then, we have

$$
\left.\mathscr{L}_{n}[\varrho(\theta) / \varrho(\psi)]\right|_{\theta=0}=\left.\varrho^{\prime}(\theta)[\varrho(\theta) / \varrho(\psi)]\right|_{\theta=0}=\left.\kappa^{-1}[\varrho(\theta) / \varrho(\psi)]\right|_{\theta=0} .
$$

Furthermore, by construction, on the cross-section $w=w_{0}, \varrho(\theta) / \varrho(\psi)=1$. Hence, along $\theta=0$, we have $\varrho(\theta) / \varrho(\psi)=\exp \kappa\left(w-w_{0}\right)$, which is regular everywhere on the generator.] The chart $w, \psi, \phi$ is well adapted to computations: $\mathscr{L}_{n} \psi=0, L_{n} \phi=0, n^{a}$ takes the form $A^{-1} n^{a} \equiv \bar{\partial}_{w}$ and $q_{a b}$ is manifestly degenerate with signature $(0,+,+)$. However, since $\mathscr{L}_{n} \theta=A \varrho(\theta), \mathscr{L}_{n} q_{a b} \neq 0$; in the chosen conformal frame, $\mathscr{I}$ is not divergence-free. We therefore perform the following rescaling:

$$
\begin{aligned}
q_{a b} \rightarrow q_{a b}^{\prime} & =\omega^{2} q_{a b} \equiv[\varrho(\psi) / \varrho(\theta)]^{2} q_{a b} \\
n^{a} \rightarrow n^{\prime a} & =\omega^{-1} n^{a} \equiv[\varrho(\theta) / \varrho(\psi)] n^{a} .
\end{aligned}
$$

Then, $\mathscr{L}_{n^{\prime}} q_{a b}^{\prime}=0 ;\left(q_{a b}^{\prime}, n^{\prime a}\right)$ corresponds to a conformal frame in which $\mathscr{I}$ is divergence-free. We can now ask if the generators of $\mathscr{I}$ are complete. Consider, first, any generator labelled by $\psi=\psi_{0}, \phi=\phi_{0} ; \psi \neq 0, \psi \neq \theta_{0}$. Along this generator, $w^{\prime}$, the affine parameter of $n^{\prime a}$ satisfying $\mathscr{L}_{n^{\prime}} w^{\prime}=A$, is related to $w$ by $d w^{\prime}=\left[\varrho\left(\psi_{0}\right) / \varrho\left(\theta\left(w, \psi_{0}\right)\right)\right] d w$. Hence, $w^{\prime}=\varrho\left(\psi_{0}\right) \int \varrho(\theta)^{-1} d w$. Since $\varrho(\theta)$ is bounded above and since $w$ ranges over $(-\infty, \infty)$ along the generator, so must $w^{\prime}$. Thus, we have shown that the vector field $n^{\prime a}$ is complete everywhere on $\mathscr{I}$ except possibly on the two generators $\psi=0$ and $\psi=\theta_{0}$. [Note that, since $\varrho(\theta)$ vanishes at $\psi=0$ and 


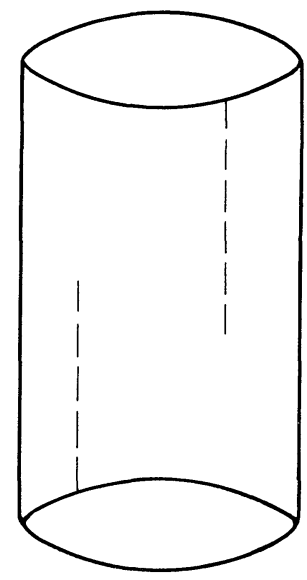

Fig. 1. The global structure of $\mathscr{I}$ of a charged $C$-metric (without nodes) representing the field of a single accelerated particle. The dashed lines are to be deleted. $\mathscr{I}$ has the topology of $S^{2} \times R$. However, two generators $(\psi=0$ and $\psi=\pi)$ are incomplete

$\psi=\theta_{0}$, so does the Killing field $\partial_{\phi}$. Hence $\psi=0$ and $\psi=\theta_{0}$ define single generators rather than a 2-dimensional submanifold of $\mathscr{I}$.] Let us now consider the generator $\psi=0$. On this generator we have: $d w^{\prime}=\left.[\varrho(\psi) / \varrho(\theta)]\right|_{\psi=0} d w=\left[\exp \kappa^{-1}\left(w_{0}-w\right)\right] d w$; i.e., $w^{\prime}=-\kappa \exp \left[\kappa^{-1}\left(w_{0}-w\right)\right]+$ constant. Consequently, as $w$ ranges from $-\infty$ to $\infty, w^{\prime}$ takes values between $-\infty$. and $\kappa$, where we have chosen the convention that $w^{\prime}=0$ iff $w=0$. Thus, the generator $\psi=0$ is in fact incomplete. An identical calculation shows that the generator $\psi=\theta_{0}$ is also incomplete. However, since $\partial \varrho(\theta) / \partial \theta=-\kappa$ at $\theta=\theta_{0},\left.[\varrho(\psi) / \varrho(\theta)]\right|_{\psi=\theta_{0}}=\exp \kappa^{-1}\left(w-w_{0}\right)$, whence $w^{\prime}$ now ranges between $-\kappa$ and $+\infty$. We are thus led to Fig. 1; the space-times under consideration are not asymptotically Minkowskian.

Next, we show that these space-times do admit gravitational as well as electromagnetic radiation. The Weyl tensor $C_{a b c d}$ of $g_{a b}$ can be easily computed from Eq. (2.10) :

$$
C_{a b c d}=\left(1 / r^{2}\right) \hat{C}_{a b c d}
$$

Thus, as is expected from general theorems [1,2], $C_{a b c d}$ vanishes on $\mathscr{I}$ and $K_{a b c d}:=\Omega^{-1} C_{a b c d}$ admits a smooth limit to $\mathscr{I}$. Set $K_{a c}=K_{a b c d} n^{b} n^{d}$. The pull-back $K_{a b}$ of $K_{a b}$ to $\mathscr{I}$ is given by:

$$
\begin{aligned}
K_{a b}= & 3 A^{2} \varrho^{2}(\theta) P\left(-\varrho(\theta)^{2} D_{a} w D_{b} w-D_{a} \theta D_{b} \theta\right. \\
& \left.+\kappa^{2} \varrho(\theta)^{2} D_{a} \phi D_{b} \phi+2 \varrho(\theta) D_{(a} \theta D_{b)} w\right),
\end{aligned}
$$

where, as before, $\left.P\right|_{\mathscr{g}}=m+2 e^{2} A x$, and, where, $D$ denotes the gradient operator on $\mathscr{I}$. Hence, the restriction to $\mathscr{I}$ of ${\underset{K}{a b}}_{a b}$ is non-zero. In the Newman-Penrose [11] notation, this implies that $\psi_{4}^{0}$ is non-zero, whence the news function cannot vanish identically on $\mathscr{I}$. In the Geroch [2] notation, $K_{a b}$ can be expressed in terms of the derivative of the news tensor, whence non-vanishing of $K_{a b}$ implies that the news tensor cannot vanish. [Newman-Penrose as well as Geroch work in conformal 
frames in which $\mathscr{I}$ is divergence-free. Hence, we should have worked in the frame $\left(q_{a b}^{\prime}, n^{\prime a}\right)$ rather than $\left(q_{a b}, n^{a}\right)$. However, since $K_{a b c d}$ has conformal weight +1 , and $n^{a}$ weight -1 , we have ${\underset{\leftarrow}{L}}_{a b}^{\prime}=[\varrho(\theta) / \varrho(\psi)]{\underset{\leftarrow}{a b}}_{a b}$. Hence $\underline{K}_{a b}^{\prime}$ is also non-zero on $\mathscr{I}$. $]$. Next, let us examine the asymptotic behavior of the electromagnetic field. From Eq. (2.2) we have:

so that

$$
F_{a b} \equiv \hat{F}_{a b}=2 e \nabla_{[a} y \nabla_{b]} w
$$

$$
F_{a b} n^{b}=A e \nabla_{a} y-A e F(y) \nabla_{a} w
$$

Hence the pull-back to $\mathscr{I}$ of $F_{a b} n^{b}$ is non-zero. In the Newman-Penrose notation this implies that the radiation field $\phi_{2}^{0}$ fails to vanish on $\mathscr{I}$.

Finally, we remark that we have examined in this section only future null infinity. The introduction of past null infinity is completely analogous.

\section{Analytic Extensions: The Flat $C$-Metric}

The class of solutions without nodes considered in Sect. 3 contains the flat metric, which corresponds to $m=e=0$. Even for this metric, our construction yields a $\mathscr{I}$ in which "two half-generators are missing". How does this come about? As one might expect, our charts cover only a part of Minkowski space-time. More generally, every space-time analysed in Sect. 3 is geodesically incomplete and can be extended analytically. In order to bring out the geometrical aspects of this extension, we shall now restrict ourselves to the flat $C$-metric and carry out the extension explicitly. While these extensions are not really necessary for metrics without nodes - we have already shown that these metrics are asymptotically flat in the desired sense - they are essential in the case of metrics with nodes to be treated in the next section.

Let $\left(\hat{M}, \hat{g}_{a b}\right)$ denote the flat $C$-space-time obtained by setting $m=e=0$ in Eq. (2.8). Using the ideas underlying the Kruskal extension of the Schwarzschild solution we set [16]:

$$
u=\exp w ; \text { and } \quad v=u^{-1}\left(\frac{y-1}{y+1}\right) .
$$

[Note: $\quad u=\exp \left(t+\int\left(y^{2}-1\right)^{-1} d y\right)$ and $v= \pm \exp \left(-t+\int\left(y^{2}-1\right)^{-1} d y\right)$. Since $m=e=0, F(y)=y^{2}-1$.] The metric $\hat{g}_{a b}$ is now given by:

$$
d \hat{s}^{2}=r^{2}\left[(y+1)^{2} d u d v+d \theta^{2}+\sin ^{2} \theta d \phi^{2}\right],
$$

where $y$ and $r$ are now to be regarded as functions of $u, v$ :

$$
y=\frac{1+u v}{1-u v} ; \quad \operatorname{Ar}=\frac{1-u v}{1-\cos \theta+u v(1+\cos \theta)} .
$$

The manifold $\hat{M}$ now corresponds to the following coordinate ranges:

$$
\theta \in[0, \pi] ; \quad \phi \in[0,2 \pi] ; \quad u>0 ; \quad \text { and } \quad \frac{\cos \theta-1}{\cos \theta+1}<u v<1
$$



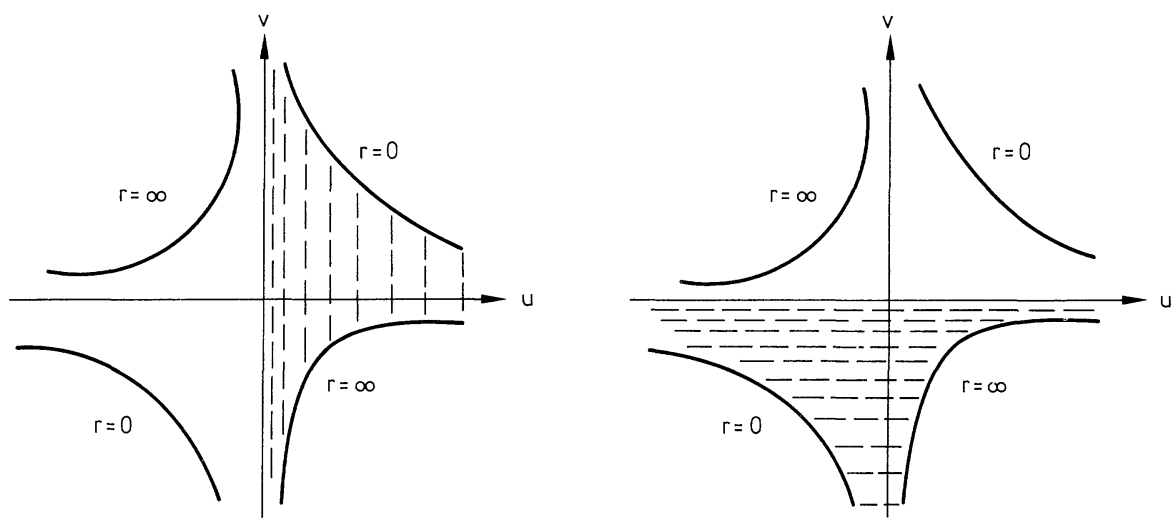

Fig. 2. A The region enclosed by the four curves $r=0$ and $r=\infty$ represents a $\theta=$ constant $\left(\neq 0 ; \neq \theta_{0}\right)$, $\phi=$ constant surface in the extended space-time $\hat{M}^{\prime}$. The lined region represents the original space-time $\hat{M}$. The curves $r=0$ represent the "particle trajectories". Thus, while $\hat{M}$ represents the field of a single "particle", $\hat{M}^{\prime}$ represents the field of two "particles". B The lined region represents the part of $\hat{M}^{\prime}$ covered by the $\tilde{w}, y, \theta, \phi$ chart. The two charts, $w, y, \phi, \phi$ and $\tilde{w}, y, \theta, \phi$ overlap in the part $u>0, v<0$ of this region. The curve $r=\infty$ at the boundary of the overlap represents the future null infinity. The second $r=\infty$ curve represents the past null infinity

[Since $m=e=0, \varrho(\theta)=\sin \theta, \theta_{0}=\pi$, and $\kappa=1$.] We can extend $\hat{M}$ by allowing $u, v$ to take any values consistent with the condition on the product $u v$ given in Eq. (4.4) (see Fig. 2A). Denote this extended manifold by $\hat{M}^{\prime}$. Note that the metric $\hat{g}_{a b}$ is well-defined everywhere on $\hat{M}^{\prime}$; the points $y=-1$ do not belong to $\hat{M}^{\prime}$. What is the relation between $\hat{M}^{\prime}$ and Minkowski space-time? Set

$$
\begin{aligned}
& \bar{x}=r \sin \theta \cos \phi, \\
& \bar{y}=r \sin \theta \sin \phi, \\
& \bar{z}=r \frac{u+v}{1-u v}, \quad \text { and } \\
& \bar{t}=r \frac{u-v}{1-u v} .
\end{aligned}
$$

Then $\hat{g}_{a b}$ takes the form:

$$
d \hat{s}^{2}=d \bar{x}^{2}+d \bar{y}^{2}+d \bar{z}^{2}-d \bar{t}^{2} .
$$

Thus, $\bar{x}, \bar{y}, \bar{z}, \bar{t}$ are the usual Cartesian coordinates. Equation (4.5) defines an imbedding of $\hat{M}^{\prime}$ into Minkowski space. Using Eqs. (4.4) and (4.5), it is easy to verify that the image of $\hat{M}^{\prime}$ is all of Minkowski space except for two lines, given by $\bar{x}=\bar{y}=0, \bar{z}^{2}-\bar{t}^{2}=1 / A^{2}$, at which $r=0$. [At these points, the coordinate system $u, v, \theta, \phi$ can not be extended due to the overall factor of $r^{2}$ in the expression (4.2) of the metric.] One could, of course, have carried out a further extension of $\hat{M}^{\prime}$ [e.g. via Eq. (4.5)] to include these lines. However, in the general case, with at least one of $m$ and $e$ non-zero, the lines $r=0$ correspond to curvature singularities. Since the present discussion of the flat $C$-metric is only a prelude to that of the general case, 
we shall treat these lines as "singularities" and refrain from carrying out a further extension.

To summarize, the manifold $\hat{M}$ covers only the region of Minkowski space defined by $\bar{z}+\bar{t}>0$; the world line $\bar{x}=\bar{y}=0, \bar{z}^{2}-\bar{t}^{2}=1 / A^{2}$ being deleted. The extended manifold $\hat{M}^{\prime}$ corresponds to all of Minkowski space except for two world lines (of constant acceleration) defined by $\bar{x}=\bar{y}=0, \bar{z}^{2}-\bar{t}^{2}=1 / A^{2}$. The conformal completion of $\hat{M}$ yielded a $\mathscr{I}$ with "two half-generators missing". We shall now show that the $\mathscr{I}$ of $\hat{M}^{\prime}$ misses only two points representing the intersection of the "singularities" with $\mathscr{I}$.

The idea is to carry out the conformal completion using, once again, $r^{-1}$ as the conformal factor. Thus, $\mathscr{I}$ will be the set of points at which $r=\infty$; i.e., $u v=(\cos \theta-1) /(\cos \theta+1)$. Unfortunately, the rescaled metric, $g_{a b}=r^{-2} \hat{g}_{a b}$, has "coordinate singularities" (in the $u, v$ chart) on $\mathscr{I}$ : since $(\mathrm{Ar})^{-1}=-\cos \theta+\mathrm{y}=0$ on $\mathscr{I}, y=-1$ at $\theta=\pi$ on $\mathscr{I}$, whence, from Eq. (4.2), $g_{a b}$ appears to be degenerate on a generator. Therefore we need two coordinate patches to cover $\mathscr{I}$. Since we have already made detailed constructions in Sect. 3 using $w, y, \theta, \phi$ as coordinates, we shall use them again for the first patch. This, as we know, covers the region $u>0$ of the $u, v$ patch [Eq. (4.4)]. To obtain the second patch, we introduce a new coordinate $\tilde{w}$ on the region defined by $v<0,(\cos \theta-1) /(\cos \theta+1)<u v<1$ (see Fig. 2B):

$$
\tilde{w}=\log (-v) .
$$

In the chart $\tilde{w}, y, \theta, \phi$ the metric $g_{a b}$ takes the form:

$$
d s^{2}=-\left(y^{2}-1\right) d \tilde{w}^{2}+2 d \tilde{w} d y+d \theta^{2}+\sin ^{2} \theta d \phi^{2}
$$

while we know, from Eq. (2.8), that in the $w, y, \theta, \phi$ chart it has the form:

$$
d s^{2}=-\left(y^{2}-1\right) d w^{2}+2 d w d y+d \theta^{2}+\sin ^{2} \theta d \phi^{2} .
$$

The two charts intersect in the region $u>0, v<0$ of the $u, v$ coordinate system and are related in a $C^{\infty}$ fashion there since $w+\tilde{w}=\log \left|\frac{y-1}{y+1}\right|$. (See Fig. 2A and 2B. Recall that $y$ cannot assume the value -1 on $\hat{M}^{\prime}$. At $y=1, u v=0$, whence these points do not belong to the region under consideration.) Each of the two patches $w, y, \theta, \phi$ and $\tilde{w}, y, \theta, \phi$ can be obviously extended to allow $r$ to take the value $r=\infty$; i.e. to include the $y=\cos \theta$ surface. Denote by $\mathscr{I}_{w}$ the " $r=\infty$ surface" in the first patch, and by $\mathscr{I}_{\tilde{w}}$ that in the second. Set $\mathscr{I}_{=} \mathscr{I}_{w} \cup \mathscr{I}_{\tilde{w}} . \mathscr{I}_{w}$ and $\mathscr{I}_{\tilde{w}}$ can be, respectively, coordinatized by $w, \theta, \phi$ and $\tilde{w}, \theta, \phi$. The analysis of Sect. 3 shows that each is topologically $S^{2} \times R$ but "misses half a generator" at $\theta=0$ as well as at $\theta=\pi$. Finally, since $w+\tilde{w}=\log \left|\frac{\cos \theta-1}{\cos \theta+1}\right|$ on $\mathscr{I}$, it follows that $\mathscr{I}_{w} \cap \mathscr{I}_{w}$ contains all of $\mathscr{I}$ except the generators $\theta=0$ and $\theta=\pi$. The metric $g_{a b}$ of Eq. (4.9) is wellbehaved everywhere on $\mathscr{I}_{w}$, and that of Eq. (4.8) on $\mathscr{I}_{w}$. On the overlap $\mathscr{I}_{w}$, the two metrics, of course, agree.

To investigate the global structure of $\mathscr{I}$, we first introduce, as in Sect. 3, the coordinate $\psi$ on $\mathscr{I}_{w}$, make $\mathscr{I}_{w}$ divergence-free using the conformal transformation of Eq. (3.8), and define the affine parameter $w^{\prime}$ on the integral curves of $n^{\prime a}$. Then 


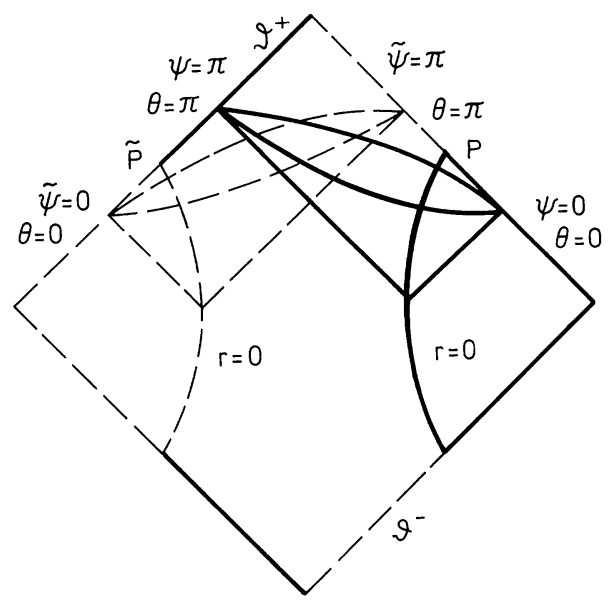

Fig. 3. The asymptotic structure of the extended flat $C$-metric. The solid lines refer to the geometrical structure arising from a single "particle trajectory" of constant acceleration, i.e. to the space-time $\hat{M}$ covered by coordinates $w, y, \theta, \phi$ while the dashed-lines refer to the new structure arising from the extension. Note that, on the past half of the generator of $\mathscr{I}^{+}$, labelled by $\psi=0$ (or, $\tilde{\psi}=\pi$ ), $\theta$ vanishes, while on the future half, $\theta=\pi$. The same situation occurs on the generator labelled by $\psi=\pi(\tilde{\psi}=0)$. Each of $\mathscr{I}_{w}$ (solid lines) and $\mathscr{I}_{\tilde{w}}$ (dashed-lines) contains a $\theta=0$ piece and a $\theta=\pi$ piece and is therefore topologically $S^{2} \times R$. The points $p$ and $\tilde{p}$ are not contained in $\mathscr{I}_{\equiv} \equiv \mathscr{I}_{w} \cup \mathscr{I}_{\tilde{w}}$

the generators of $\mathscr{I}_{w}$ can be labelled by $\psi$ and $\phi$, and $w^{\prime}$ takes values in $(-\infty, \infty)$ on all generators except $\psi=0$ and $\psi=\pi$, in $]-\infty, 1[$ on the generator $\psi=0$, and in $]-1, \infty\left[\right.$ on $\psi=\pi$ (see Fig. 1 ). Next, consider $\mathscr{I}_{\tilde{w}}$. Since, on the intersection $\mathscr{I}_{w} \cap \mathscr{I}_{w}, d w+d \tilde{w}=2 d \theta / \sin \theta$ [Eq. (3.6) with $\varrho(\theta)=\sin \theta$ ], the function $\tilde{\psi}=\pi-\psi$ satisfies, on $\mathscr{I}_{w} \cap \mathscr{I}_{\tilde{w}}, L_{n} \tilde{\psi}=0$ and $d \tilde{\psi} / \sin \tilde{\psi}=-d \tilde{w}+d \theta / \sin \theta$. Hence, we can label the generators of $\mathscr{I}_{\tilde{w}}$ by $\tilde{\psi}, \phi$ and express the pull-back of the space-time metric (4.8) as $d s^{2}=\left(\sin ^{2} \theta / \sin ^{2} \tilde{\psi}\right)\left(d \tilde{\psi}^{2}+\sin ^{2} \tilde{\psi} d \phi^{2}\right)$. Thus, (the extension to $\mathscr{I}_{\tilde{w}}$ of) $\tilde{\psi}$ serves the same purpose on $\mathscr{I}_{\tilde{w}}$ as $\psi$ does on $\mathscr{I}_{w}$. Consequently, we can repeat the procedure of Sect. 3 to introduce a conformal factor which makes $\mathscr{I}_{\tilde{w}}$ divergencefree, and an affine parameter $\tilde{w}^{\prime}$ along $n^{\prime a}$. This $\tilde{w}^{\prime}$ ranges between $(-\infty, \infty)$ on all generators of $\mathscr{I}_{\tilde{w}}$ except $\tilde{\psi}=0$ and $\tilde{\psi}=\pi$, over $]-\infty, 1[$ on the generator $\tilde{\psi}=0$, and over $]-1, \infty[$ on $\tilde{\psi}=\pi$. (Note that $\tilde{\psi}=0$ corresponds to $\psi=\pi$, and $\tilde{\psi}=\pi$ to $\psi=0$.) Since $w^{\prime}$ and $\tilde{w}^{\prime}$ are both affine parameters along $n^{\prime a}$ in $\mathscr{I}_{w} \cap \mathscr{I}_{\tilde{w}}$, we have $\tilde{w}^{\prime}=w^{\prime}+\alpha(\psi, \phi)$ for some smooth function $\alpha$. A detailed examination shows that $\operatorname{Lim} \alpha(\psi, \phi)=-2$ and $\operatorname{Lim} \alpha(\psi, \phi)=2$. Hence, if we use, say, $w^{\prime}$ as the affine $\psi \rightarrow 0$

parameter along $n^{\prime a}$ on all of $\mathscr{I}$, we find that it assumes all values between $-\infty$ and $\infty$ except the value $w^{\prime}=1$ on the generator $\psi=0$ and $w^{\prime}=-1$ on $\psi=\pi$. Thus $\mathscr{I}$ now "misses only two points".

In the final picture, as presented here, $u, v$ coordinates do not play any direct role; they are used only as a stepping stone to introduce the $\tilde{w}$-patch and to relate $\tilde{w}$ with $w$ and $y$. Each of the $w$ and $\tilde{w}$ patches leads to all of $\mathscr{I}$ except for two halfgenerators. The two patches are complementary; the half-generators missing in one patch appear in the other. The geometrical reason behind this phenomenon is made clear in Fig. 3. 


\section{Vacuum Solutions with Nodes}

We can now analyze the metrics with nodes. Since we have already demonstrated the existence of electrovac solutions which are asymptotically empty and flat, we restrict ourselves in this section to vacuum metrics $(e=0)$. Thus, $F$ and $G$ appearing in Eq. (2.2) are now cubics (see Fig. 4). In order to endow the $x-z$ submanifold with the topology of $S^{2}$, we shall again assume that $G$ has at least two real roots; i.e. $G$ (and $F$ ) has exactly three real roots. This restricts the parameters by $m A<(27)^{-1 / 2}$.

Consider the manifold $\hat{M}$ defined by Eq. (2.8) via coordinates $w, y, \theta, \phi$. The metric $\hat{g}_{a b}$ now has a nodal singularity at $\theta=\theta_{0}$. We can, nonetheless, repeat the construction in Sect. 3 and define $\mathscr{I}_{w}$ as the surface " $r=\infty$ ", i.e., $x+y=0$. As before, $\mathscr{I}_{w}$ is a 3 -manifold with topology $S^{2} \times R$, coordinatized by $w, \theta, \phi$. However, the rescaled metric $g_{a b}=r^{-2} \hat{g}_{a b}$ inherits a nodal singularity from $\hat{g}_{a b}$, which continues to exist at $\theta=\theta_{0}$ on $\mathscr{I}_{w}$ as well ${ }^{5}$. Since the definition of asymptotic flatness requires that $g_{a b}$ be regular everywhere on $\mathscr{I}$, one is forced to delete the generator $\theta=\theta_{0}$ from $\mathscr{I}_{w}$. The resulting 3-manifold is topologically $R^{3}$, rather than $S^{2} \times R$, whence $\left(\hat{M}, \hat{g}_{a b}\right)$ is not asymptotically empty and flat in the sense of Sect. 2 . However, the discussion in Sect. 4 suggests that one may be able to "fill in the missing generator" by carrying out an analytic extension of $\left(\hat{M}, \hat{g}_{a b}\right)$ before the conformal completion. The idea is best explained in terms of Fig. 3: since the "bottom half" of the generator labelled by $\theta=\pi$ in the $w, y, \theta, \phi$ patch (i.e. in the field of the first particle, represented by a solid line) is labelled by $\theta=0$ in the $\tilde{w}, y, \theta, \phi$ patch (i.e. in the field of the second particle, represented by a dashed line), and since the metric $g_{a b}$, in the present case, is regular at $\theta=0$, one might expect that an analytical continuation similar to the one in Sect. 4 will lead to a $\mathscr{I}$ which is $S^{2} \times R$ in which only the two half-generators labelled by $\theta=\pi$ are missing. We shall now show that this expectation is correct.

Define, on the region $y<y_{3}$ of $\hat{M}$, new coordinates $u$ and $v$ by [16]

$$
u=\exp (c w) ; \quad v=u^{-1} \frac{y-y_{2}}{\left(y-y_{1}\right)^{n_{1}}\left(y_{3}-y\right)^{n_{3}}},
$$

where

$$
2 c=d F /\left.d y\right|_{y=y_{2}} ; \quad 0<n_{1}=\left(y_{3}-y_{2}\right) /\left(y_{3}-y_{1}\right) ; \quad 0<n_{3}=\left(y_{2}-y_{1}\right) /\left(y_{3}-y_{1}\right) .
$$

[Equation (5.1) is more complicated than Eq. (4.1) because $F$ is now cubic, rather than quadratic, in $y$. The roots $y_{i}$ of $F(y)$ are related to the roots $x_{i}$ of $G(x)$ by $y_{i}=-x_{i}$ since $F(y)=-G(-y)$. It follows from Fig. 4 that $y_{1}<y_{2}<y_{3}$. Note that since $0<r<\infty$ on $\hat{M}, y$ cannot attain the value $y=y_{1}$ on $\hat{M}$.] In the $u-v$ chart the rescaled metric $g_{a b}=r^{-2} \hat{g}_{a b}$ is given by

$$
d s^{2}=\frac{-2 m A}{c^{2}}\left(y-y_{1}\right)^{n_{1}+1}\left(y_{3}-y\right)^{n_{3}+1} d u d v+d \theta^{2}+\kappa^{2} \varrho(\theta)^{2} d \phi^{2},
$$

5 This is why a Newman-Unti coordinate system - global in angular variables - in which the metric is regular, i.e., say $C^{2}$, is unlikely to exist in the asymptotic region of the field of a single particle 


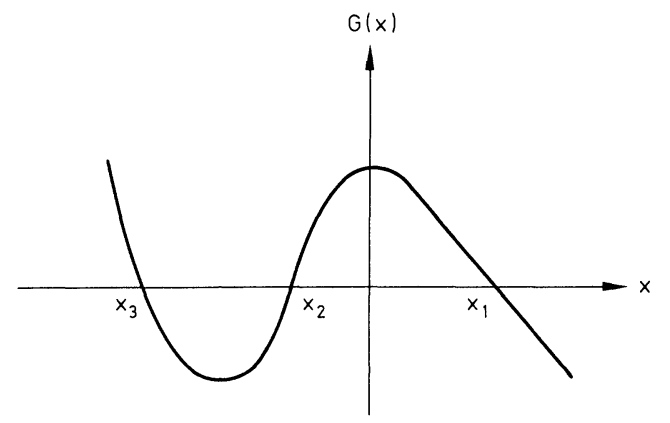

Fig. 4. The graph of $G(x)$ in the case $e=0, m A<(27)^{-1 / 2} . G(x)$ now admits three distinct roots, $x_{1}>x_{2}>x_{3}$. We restrict ourselves to the region $\left[x_{2}, x_{1}\right]$ in which $G(x)$ is non-negative

where $y$ is now to be regarded as a function of $u$ and $v$ :

$$
\frac{1}{c} \log |u v|=2 \int F(y)^{-1} d y .
$$

The metric is clearly well-defined for all values of $u$ and $v$ except those which correspond to $y=y_{1}$ and $y=y_{3}$. The region $y<y_{3}$ of $\hat{M}$ is given by

$$
\theta \in\left[0, \theta_{0}\right] ; \quad \phi \in[0,2 \pi] ; \quad u>0 ; \quad \frac{x_{2}-x}{\left(x_{1}-x\right)^{n_{1}}\left(x-x_{3}\right)^{n_{3}}}<u v<\infty
$$

where $x=\int_{0}^{\theta} \varrho(\theta) d \theta+x_{2}$. We shall denote by $\hat{M}^{\prime}$ the manifold obtained by replacing the requirement $u>0$ by $-\infty<u<\infty$.

We now wish to consider the surface " $r=\infty$ " defined by $u v=\left(x_{2}-x\right) /\left(x_{1}-x\right)^{n_{1}}\left(x-x_{3}\right)^{n_{3}}$. On this surface $x+y=0$, and hence $y$ can take the value $y=y_{1}$. At $y=y_{1}$, the metric of Eq. (5.2a) appears to be degenerate. (Note that since $x \in\left[x_{2}, x_{1}\right], y$ is constrained to the interval $\left[y_{1}, y_{2}\right]$ on this surface and can not attain the value $y=y_{3}$.) This situation is completely analogous to that in Sect. 4. We therefore introduce, as before, a new coordinate $\tilde{w}$ on the region $v<0$ of $\hat{M}^{\prime}$ by:

$$
\tilde{w}=c^{-1} \log (-v)
$$

and work in the region $u>0, v<0$ of the $u-v$ plane, the intersection of the $w, y, \theta, \phi$ and $\tilde{w}, y, \theta, \phi$ charts. In this intersection, $w$ and $\tilde{w}$ are smoothly related: $w+\tilde{w}$ $=c^{-1} \log \left|\left(y-y_{2}\right) /\left(y-y_{1}\right)^{n_{1}}\left(y-y_{3}\right)^{n_{3}}\right|$ and the metric takes the form:

$$
\begin{aligned}
d s^{2} & =-F(y) d w^{2}+2 d w d y+d \theta^{2}+\kappa^{2} \varrho(\theta)^{2} d \phi^{2}, \\
& =-F(y) d \tilde{w}^{2}+2 d \tilde{w} d y+d \theta^{2}+\kappa^{2} \varrho(\theta)^{2} d \phi^{2} .
\end{aligned}
$$

Therefore, both charts can be extended to include the 3-surfaces " $r=\infty$ " defined by $x+y \equiv x_{2}+\int \varrho(\theta) d \theta+y=0$. Let $\mathscr{I}_{w}$ and $\mathscr{I}_{\tilde{w}}$ denote, respectively, these 3 -surfaces in the $w, y, \theta, \phi$ and $\tilde{w}, y, \theta, \phi$ charts. Each is a 3-manifold, topologically $S^{2} \times R$. As in Sect. 3 , we can coordinatize $\mathscr{I}_{w}$ by $w, \psi, \phi$ and $\mathscr{I}_{\tilde{w}}$ by $\tilde{w}, \tilde{\psi}, \phi$. Then, on $\mathscr{I}_{w}, \psi$ satisfies Eq. (3.6) and the condition $L_{n} \psi=0$, and, on $\mathscr{I}_{\tilde{w}}, \tilde{\psi}$ satisfies the tilde- 


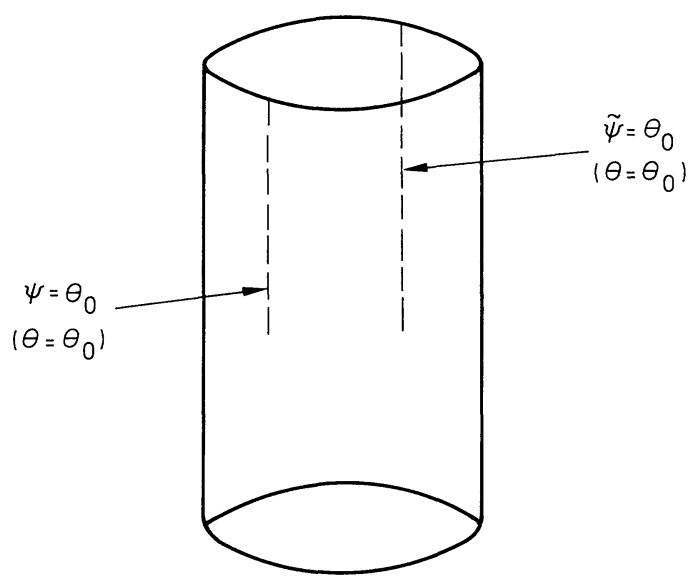

Fig. 5. The global structure of $\mathscr{I}$ of a vacuum $C$-metric. The dashed lines are to be deleted. $\mathscr{I}$ is topologically $S^{2} \times R$. The generators labelled by $\psi=\theta_{0}$ and $\tilde{\psi}=\theta_{0}$ are incomplete due to nodal singularities. Note that, in contrast to the situation in Fig. 1, both generators are incomplete in the future

version of Eq. (3.6) and $\mathscr{L}_{n} \tilde{\psi}=0$. What is the intersection of $\mathscr{I}_{w}$ and $\mathscr{I}_{\tilde{w}}$ ? Although $y$ cannot assume the values $y=y_{1}$ or $y=y_{2}$ on the region $u>0, v<0$ of $\hat{M}$, it can take these values on $\mathscr{I}_{w}$ and $\mathscr{I}_{\tilde{w}}$ along the generators labelled by $\theta=0$ and $\theta=\theta_{0}$. Consequently, $\mathscr{I}_{w} \cap \mathscr{I}_{\tilde{w}}$ contains all of $\mathscr{I}_{w} \cup \mathscr{I}_{\tilde{w}}$ except for the generators labelled by $\psi=0$ and $\psi=\theta_{0}$ of $\mathscr{I}_{w}$ and the generators labelled by $\tilde{\psi}=0$ and $\tilde{\psi}=\theta_{0}$ on $\mathscr{I}_{\tilde{w}}$. On the intersection, $\mathscr{I}_{w} \cap \mathscr{I}_{\tilde{w}}$, one can ask for the relation between $\psi$ and $\tilde{\psi}$. Using the fact that $\psi$ satisfies Eq. (3.6) and $\tilde{\psi}$ its tilde-version, and the relation between $w$ and $\tilde{w}$, it is easy to show that, on $\mathscr{I}_{w} \cap \mathscr{I}_{\tilde{w}}$, we have: $-d \psi / \varrho(\psi)=d \tilde{\psi} / \varrho(\tilde{\psi})$. The properties of $\varrho$ now imply that $\psi$ and $\tilde{\psi}$ are smoothly related and satisfy: $\operatorname{Lim}_{\tilde{\psi} \rightarrow 0} \psi=\theta_{0}$ and $\operatorname{Lim}_{\tilde{\psi} \rightarrow \theta_{0}} \psi=0$. (Recall, in the flat $C$-metric, we could arrange matters so that $\tilde{\psi}=\pi-\psi$. In the general case, we can, apparently, make statements only about limits.) Using these limits, we can extend the function $\psi$ to all of $\mathscr{I}_{w} \cup \mathscr{I}_{\tilde{w}}$. Next, consider the points on $\mathscr{I}_{w} \cup \mathscr{I}_{\tilde{w}}$ labelled by $\theta=\theta_{0}$. These correspond to the points $\psi=\theta_{0}$ in the first chart and to $\tilde{\psi}=\theta_{0}$ in the second. Delete these points from $\mathscr{I}_{w} \cup \mathscr{I}_{\tilde{w}}$ and denote the resulting 3-manifold by $\mathscr{I}$. We claim that $\mathscr{I}$ is topologically $S^{2} \times R$ : in terms of the function $\psi$ which takes values in $\left[0, \theta_{0}\right]$ we have deleted only "half-generators" at $\psi=0$ and $\psi=\theta_{0}$. More precisely, since the points at which $\theta=\theta_{0}$ do not belong

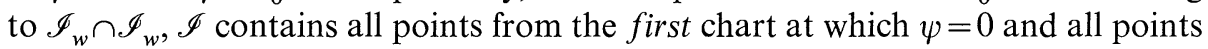
from the second chart at which $\tilde{\psi}=0$, i.e. (the extended function) $\psi=\theta_{0}$. This 3-manifold, $\mathscr{I}$, is the required null-boundary. Next, consider the metric $g_{a b}$ of Eq. (5.5). Since $\theta$ never assumes the value $\theta_{0}$ on $\mathscr{I}$-i.e. since all zeros of $\varrho(\theta)$ on $\mathscr{I}$ occur at $\theta=0$ - the metric is everywhere regular on $\mathscr{I}$. Finally, condition iv) in the definition of asymptotic flatness is trivially satisfied since $\hat{R}_{a b}$, the Ricci tensor of $\hat{g}_{a b}$, vanishes everywhere on $\hat{M}^{\prime}$. Thus, $\left(\hat{M}^{\prime}, \hat{g}_{a b}\right)$ is asymptotically empty and flat at null infinity. That the radiation field (i.e. $K_{a b}$ ) does not vanish identically on $\mathscr{I}$ follows immediately from Eq. (3.10); since it suffices to show that there exists an 
open region of $\mathscr{I}$ on which $\mathbb{K}_{a b}$ is non-zero, one can restrict oneself to the $w, y, \theta, \phi$ patch.

It is straightforward to analyse the global structure of $\mathscr{I}$ by introducing a divergence-free conformal frame as in Sect. 3. All generators, except those labelled by $\psi=0$ and $\psi=\theta_{0}$, are complete. In contrast to Fig. 1 , however, along both incomplete generators, the divergence-free vector field $n^{\prime a}$ is complete in the past (see Fig. 5). If we had chosen the constant $\kappa$ such that the metric of Eq. (2.5) were regular at $\theta=\theta_{0}$, in place of $\theta=0, n^{\prime a}$ would have been complete in the future along both generators. In either case, $\left(\hat{M}^{\prime}, \hat{g}_{a b}\right)$ fails to be asymptotically Minkowskian; with the choice of $\kappa$ made in Sect. 2 the node appears as a "strut" between the two particles, while the other choice yields "strings" which go out to (spatial) infinity from each particle.

\section{Discussion}

There exist in the literature $[8,12,13]$ a number of remarks concerning the nature of singularities of the $C$-metric. However, since a detailed demonstration that the underlying space-time is asymptotically flat at null infinity was not available, these statements have remained somewhat vague. Using the explicit construction of $\mathscr{I}$ presented in the previous three sections, one can now hope to undertake a thorough investigation and resolve the issue completely. Here, we shall merely outline the final picture, as we presently see it.

Consider first the case when the function $F(y)$ appearing in the expression of the metric has precisely two real roots, and at least one of the parameters $e$ and $m$ is non-zero. Then, the situation closely resembles the one in the case of the flat $C$-metric; the only essential difference is that lines $r=0$ now correspond to curvature singularities. The 2 -dimensional sub-manifolds, defined by $\theta=$ constant $\left(\neq 0, \neq \theta_{0}\right)$ and $\phi=$ constant, of the extended space-time $\hat{M}^{\prime}$ can again be represented by Fig. $2 \mathrm{~A}$. Each of these sub-manifolds is time-like and intersects $\mathscr{I}^{+}$ in a space-like ${ }^{6}$ curve $r=\infty$ in the $u>0, v<0$ quadrant. It is clear from the figure that the solution does not contain any black holes : all partial Cauchy surfaces are wholly contained in the causal past [in the completed space-time $\left.\left(M, g_{a b}\right)\right]$ of $\mathscr{I}^{+}$. Furthermore, since the curves $u=$ constant or $v=$ constant are null (in fact, null geodesics!), it is also clear from the figure that both singularities (at $r=0$ ) are naked; both are visible from $\mathscr{I}^{+}$.

The situation w.r.t. the vacuum metrics discussed in Sect. 5 is much more complicated since, in this case, $F(y)$ is a cubic. Denote, as before, the roots of $F(y)$ by $y_{1}, y_{2}, y_{3}$ with $y_{1}<y_{2}<y_{3}$. The $u, v$ coordinates, introduced in Sect. 5, cover only the $y<y_{3}$ region of the space-time. Since $x$ is constrained to lie in $\left[x_{2}, x_{1}\right]$, the singularities, $r=0$, correspond to $y=\infty$. These are therefore not contained in the $u, v$ chart. In fact, the metric $g_{a b}$ appears to be degenerate in this chart already at $y=y_{3}$ [Eq. (5.2)] and one needs to carry out another Kruskal-type extension to include the region $y \geqq y_{3}$. When this extension is carried out, one is left with the space-time diagram of Fig. 6. Since the norm, $-r^{2} F(y)$, of the Killing field $\partial_{t}$ is positive for all $y>y_{3}$, the Killing field is space-like in the extended region. Since the

6 In the Penrose diagrams in [8], this intersection appears as null 


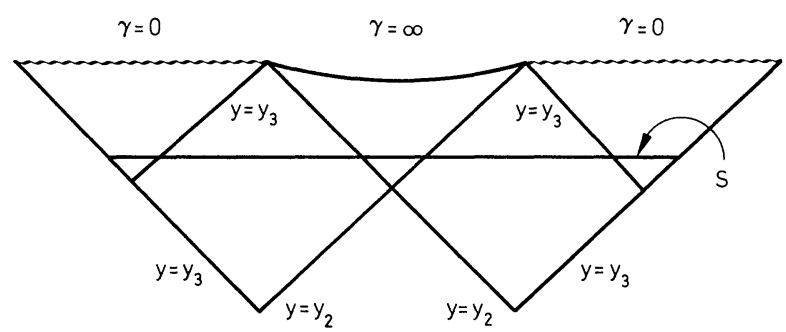

Fig. 6. The space-time diagram in the case $e=0, m A<(27)^{-1 / 2}$. [The $\theta=$ constant $\left(\neq 0, \neq \theta_{0}\right)$, $\phi=$ constant 2-plane.] The curve $r=\infty$ represents the intersection of $\mathscr{I}^{+}$with the given 2-plane. The curvature singularities $r=0$ are hidden behind the horizon $y=y_{3} . S$ is a partial Cauchy surface. $S-S \cap J^{-}\left(\mathscr{I}^{+}\right)$has two disconnected components

$r=$ constant curves in the 2-dimensional sub-manifold under consideration represent the Killing trajectories, the two singularities, $r=0$, are now "space-like". It is clear from Fig. 6 that they are hidden behind the horizons $y=y_{3}$. One can now draw partial Cauchy surfaces $S$ such that in the completed space-time $\left(M, g_{a b}\right)$, $S-S J^{-}\left(\mathscr{I}^{+}\right)$is non-empty and has two disconnected regions. In this sense, one may say that the space-time contains two black-holes. (The interpretation may appear to be ambiguous because we have focussed only on the $\theta=$ constant, $\phi=$ constant 2-surfaces. However, it is not difficult to argue that if the space-time admits a future-directed, causal curve which begins at one of the two singularities and ends on $\mathscr{I}^{+}$, then, there must also exist such a curve lying wholly in one of these 2-surfaces.) Finally, as in the Schwarzschild-Kruskal picture, we can analytically continue the space-time to include two additional "past" singularities, which would be white holes.

Thus, the overall status of singularities is essentially the same as was expected by various authors; our discussion only makes their remarks more concrete.

There is, however, a significant point concerning the black hole interpretation which, to our knowledge, has not been discussed in the literature. Normally, blackholes are defined only if the underlying space-time is asymptotically Minkowskian [14]. In the present case, $\left(\hat{M}^{\prime}, \hat{g}_{a b}\right)$ is only asymptotically empty and flat at null infinity. Note that the completeness of the generators of $\mathscr{I}^{+}$is an essential requirement in this context: if we drop this requirement, one would conclude that even Minkowski space contains a black-hole! In the case of the $C$-metric, the generators can not be complete: the isometry structure of the metric and the asymptotic behavior of the Weyl tensor themselves imply that the space-time can not be asymptotically Minkowskian [15]. Physically, the reason lies not in the nodal problems, but simply in the fact that, due to the requirement of regularity, the points of "intersection" of the curvature singularities with $\mathscr{I}^{+}$must be deleted. Hence, in the strict sense of the term, one can not even ask if the $C$-metric contains black holes! This occurs essentially because the standard definition of black holes is geared only to isolated systems and seems to exclude apriori the possibility of constantly accelerated black holes whose "trajectories" must originate on $\mathscr{I}^{-}$and end on $\mathscr{I}^{+}$. Is it possible to obtain a simple, useful generalization of the definition? 
After this work was completed and written, we learnt [16] that Schmidt has shown that certain Einstein-Rosen waves are also asymptotically empty and flat at null infinity.

Acknowledgements. We are grateful to Martin Walker for introducing us to the basic properties of the $C$-metric. Thanks are also due to the members of the Mathematical Physics group at Clermont, and in particular to Anne Magnon-Ashtekar, for their comments and suggestions. This work was completed while one of us (T.D) was a visitor at the Laboratoire de Physique Corpusculaire, Université de Clermont-Fd. He thanks the director of the Lab, D. Isabelle, for hospitality. The other author (AA) thanks R. Geroch for suggesting improvements in the manuscript and Roger Penrose for useful discussions.

\section{References}

1. Penrose, R. : Phys. Rev. Lett. 10, 66 (1963); Proc. R. Soc. (London) A 284, 159 (1965)

2. Geroch, R.: In: Asymptotic structure of space-time. Esposito, P., Witten, L. (eds.). New York: Plenum 1977

3. Lerner, D., Porter, J.R. : J. Math. Phys. 15, 1416 (1974)

4. Geroch, R., Xanthopoulos, B.C.: J. Math. Phys. 19, 714 (1978)

5. Friedrich, H.: In: Proceedings of the third gregynog workshop on gravitational radiation theory. Walker, M. (ed.). Max-Planck-Institute Green Report No. 204 (1979)

6. Levi-Civita, T.: Atti Accad. Nazl. Lincei., Rend. 27, 343 (1918)

7. Newman, E.T., Tamburino, L.: J. Math. Phys. 2, 667 (1961); Robinson, I., Trautman, A. : Proc. R. Soc. (London) A 265, 463 (1962); Ehlers, J., Kundt, W. : In : Gravitation, an introduction to current research, Witten, L. (ed.). New York: Wiley 1962

8. Kinnersley, W., Walker, M.: Phys. Rev. D2, 1359 (1970); In: Lecture notes in Physics, Vol. 14, Berlin, Heidelberg, New York: Springer 1972

9. Plebanski, J.F., Demianski, M.: Ann. Phys. 98, 98 (1976)

10. Farhoosh, H., Zimmerman, R.L. : J. Math. Phys. 20, 2272 (1979)

11. Newman, E.T., Penrose, R.: Proc. R. Soc. (London) A 305, 175 (1968)

12. Ernst, F.J.: J. Math. Phys. 17, 515 (1976); 19, 1986 (1978)

13. Dray, T., Walker, M.: Lett. Math. Phys. 4, 15 (1980)

14. Geroch, R., Horowitz, G.T.: Phys. Rev. Lett. 40, 203 (1978)

15. Ashtekar, A., Xanthopoulos, B.C.: J. Math. Phys. 19, 2216 (1978)

16. Walker, M.: J. Math. Phys. 2, 2280 (1970)

17. Schmidt, B.G. : Private communication to A. Ashtekar (1980)

Communicated by A. Jaffe

Received June 20, 1980; in revised form September 15, 1980 
